\title{
Managing Intracranial Hemorrhage in Patients with a Durable Continuous Flow Left Ventricular Assist Device
}

Citation: Jack G. et al. (2019)

"Managing Intracranial

Hemorrhage in Patients with a Durable Continuous Flow Left Ventricular Assist Device."

The VAD Journal, 5. doi:

https://doi.org/10.13023/VAD.201

$\underline{9.08}$

Editor-in-Chief: Maya Guglin, University of Kentucky

Received: June 8, 2019

Accepted: July 11, 2019

Published: July 12, 2019

(c) 2019 The Author(s). This is an open access article published under the terms of the Creative Commons Attribution-

NonCommercial 4.0 International License

(https://creativecommons.org/lice nses/by-nc/4.0/), which permits unrestricted non-commercial use, distribution, and reproduction in any medium, provided that the original author(s) and the publication source are credited.

Funding: Not applicable

Competing interests: Jason $\mathrm{N}$. Katz received consultant fees from Abbott

\section{Godly Jack1, Phil Barker², Ryan Searcy², and Jason N. Katz}

${ }^{1}$ Department of Internal Medicine, University of North Carolina School of Medicine, Chapel Hill, NC

${ }^{2}$ University of North Carolina School of Medicine, Chapel Hill, NC

${ }^{3}$ Division of Cardiology, University of North Carolina School of Medicine, Chapel Hill, NC

*Corresponding author: katzj@med.unc.edu

\section{Abstract}

\section{Background}

While intracranial hemorrhage $(\mathrm{ICH})$ is a known complication of left ventricular assist device (LVAD) support, and is associated with high morbidity and mortality, optimal care pathways have neither been elucidated nor reported. We describe management of LVAD patients following $\mathrm{ICH}$, with a focus on anticoagulation, operative interventions, care team designation, complications, and outcomes.

\section{Methods}

We retrospectively reviewed all durable continuous-flow LVAD implantations at our academic medical center from January 2007 to July 2018. Patients who experienced ICH after LVAD were identified. We defined baseline and ICH characteristics, medical and surgical interventions, care teams, and outcomes including death, device thrombosis, ischemic stroke, and hemorrhage expansion. 


\section{Results}

A total of 321 patients underwent LVAD implantation during the study period, and 27 (8\%) developed ICH (17 intraparenchymal, 7 subdural, 2 subarachnoid, 1 intraventricular) while on support. Twenty-five were anticoagulated at onset of bleed. Of those, 13 were managed with immediate cessation of anticoagulation and administration of reversal products (Group A). Group A had a median of 6 days off anticoagulation and 60 days of follow up with 1 patient $(8 \%)$ developing device thrombosis at day $8,1(8 \%)$ developing subsequent ischemic stroke at day 14 , and $4(31 \%)$ with ICH expansion. Seven patients had anticoagulation stopped at onset of bleed without administration of reversal products (Group B). With a median of 2 days off anticoagulation and 2 days of follow up, no patients in Group B developed ischemic stroke or device thrombosis while $1(14 \%)$ had ICH expansion. Five patients had anticoagulation continued at onset of bleed (Group C) with a median follow up of 330 days. One (20\%) developed device thrombosis at day 5 while $2(40 \%)$ developed $\mathrm{ICH}$ expansion. Four patients with subdural hemorrhage underwent Burr hole drainage with all 4 surviving to discharge. Two patients with intraparenchymal hemorrhage underwent open craniotomy with neither surviving to discharge. An interdisciplinary discussion occurred in all cases. Following $\mathrm{ICH}$, only one-third of patients in the study survived to 6 months.

\section{Conclusion}

LVAD patients who experience an ICH have variable outcomes. Their care is multidisciplinary and can involve operative intervention. The discontinuation and reversal of anticoagulation is generally well-tolerated, with a low risk for early device thrombosis. Like for many hemorrhagic complications of LVADs, ICH often persists or worsens. Additional investigation is needed to elucidate the most optimal management strategies.

Keywords: LVAD, intracranial hemorrhage, anticoagulation

\section{Introduction}

Though chronic heart failure is associated with substantial morbidity and mortality, the use of contemporary left ventricular assist devices (LVADs) has resulted in significant improvements among eligible patients (1-4). With that being said, there is still risk associated with device use. One well-recognized complication is that of bleeding, tied at least in part to the need for systemic anticoagulation. One potentially devastating bleeding event is that of intracranial hemorrhage (ICH). Those who develop ICH on LVAD support have markedly worse survival, and hemorrhagic strokes carry a considerably greater liability for LVAD patients than ischemic events $(5,6)$.

Although studies have identified risk factors associated with the development of ICH in LVAD patients, the actual management of this complication has been largely overlooked $(7,8)$. Treatment practices have been infrequently described and have been limited to small observational reports, and there are currently no guidelines for the management of $\mathrm{ICH}$ in this patient population. Additionally, the INTERMACS database, which collects clinical data including bleeding complications for LVAD patients, notably excludes details on the management of 
$\mathrm{ICH}$ (9). For these reasons, treatment of ICH complicating LVAD use has been largely institution-dependent and driven by clinician experience and preference.

The current study was conducted in order to describe the management of LVAD patients following $\mathrm{ICH}$, with a particular focus on anticoagulation, operative intervention, resource utilization, care team dynamics, complications, and clinical outcomes.

\section{Methods}

This study was reviewed and approved by the University of North Carolina (UNC) Institutional Review Board. Patients who received a continuous-flow, durable LVAD were identified using the UNC Mechanical Heart Program VAD tracking sheet which includes all patients at our institution who have received an intracorporeal mechanical circulatory support device since January 2007 . We performed a thorough medical record review of those patients who underwent placement of a Jarvik 2000 (Jarvik Heart, Inc., New York, NY), HeartMate II (Thoratec, Pleasanton, CA), HeartMate III (Abbott, Chicago, IL) or HVAD (Medtronic, Dublin, Ireland) from January 2007 to July 2018 in order to identify those who had developed ICH following device implantation.

Data that were systematically abstracted from the electronic medical record included patient demographics (age, gender, and race) LVAD type, ICH type (IPH, intraparenchymal hemorrhage; SDH, subdural hemorrhage; SAH, subarachnoid hemorrhage; or IVH, intraventricular hemorrhage), days post-LVAD implantation, setting at $\mathrm{ICH}$ onset, mechanism of $\mathrm{ICH}$, medical decision-making strategies (including management of antiplatelet and anticoagulation regimens, operative management including placement of Burr hole or open craniotomy), complications (including device thrombosis, ischemic stroke, and worsening bleed), resource consumption (including specialty care consultation and hospital unit placement), and mortality. Size of ICH was also calculated for IPH only (using the formula ABC divided by 2 where $A=\max$ height, $B=$ max length, $C=$ max depth). Location of bleed was collected for IPH and SAH (i.e. frontal, parietal, temporal, occipital, basal ganglia, or brainstem).

Those patients who were anticoagulated at the time of bleed were divided into three groups based on their anticoagulation management strategy: those who had anticoagulation immediately held with administration of reversal products (Group A), those who had anticoagulation immediately held without administration of reversal products (Group B), and those who had anticoagulation continued (Group C). Complication rates and survival outcomes were calculated for these three groups and stratified by type of hemorrhage.

\section{Results}

\section{Study Population}

From January 2007 to July 2018, 321 patients at our institution underwent placement of a durable, continuous-flow, intracorporeal LVAD, with 27 (8\%) subsequently developing ICH while on mechanical support. The median time between LVAD implantation and ICH was 187 days (range $=4-1739$ days). Among those who developed $\mathrm{ICH}$, the average age at time of bleed was 63.1 
years with the vast majority of patients being Caucasian or African American males with a HeartMate II (Table 1).

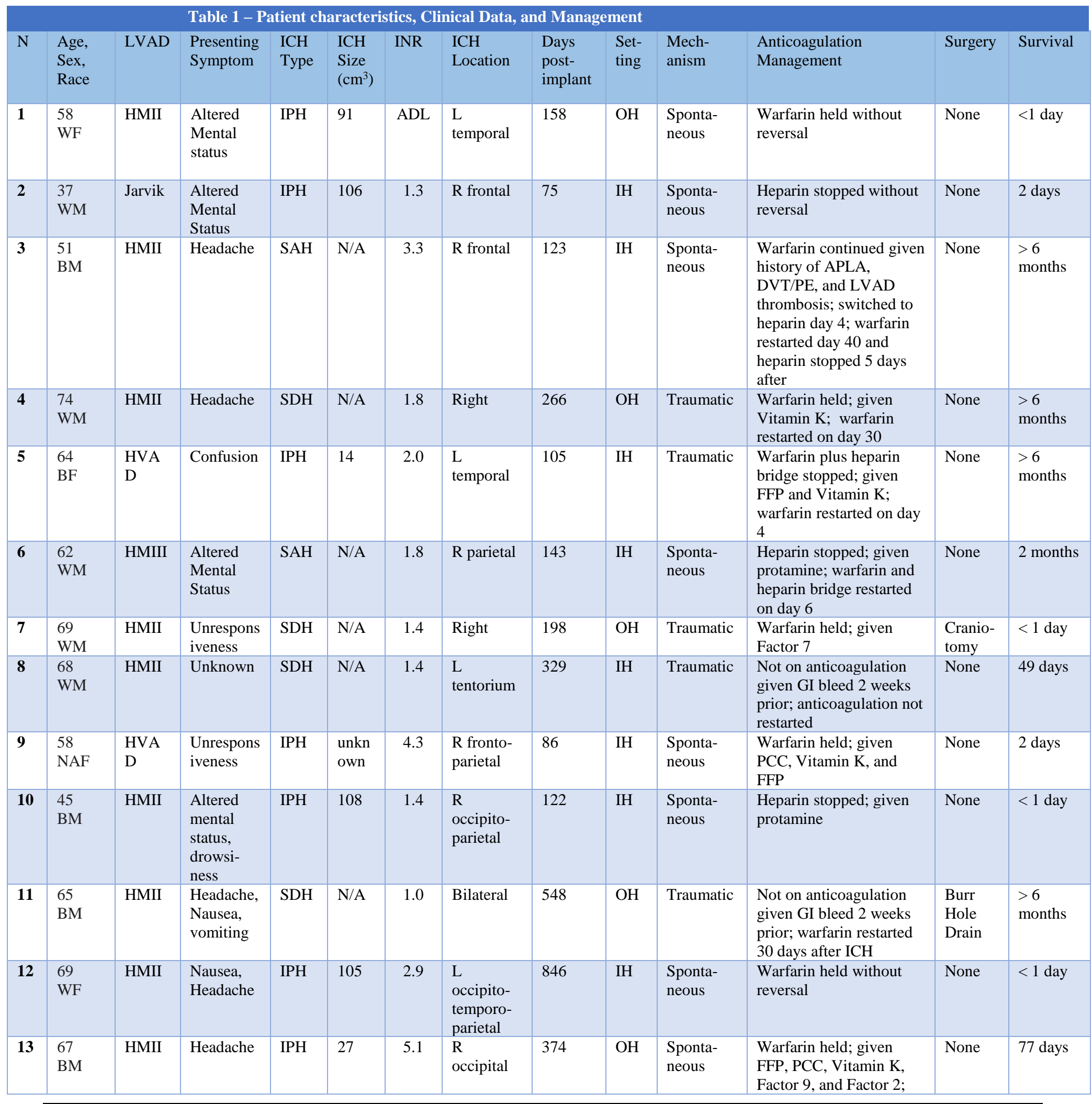




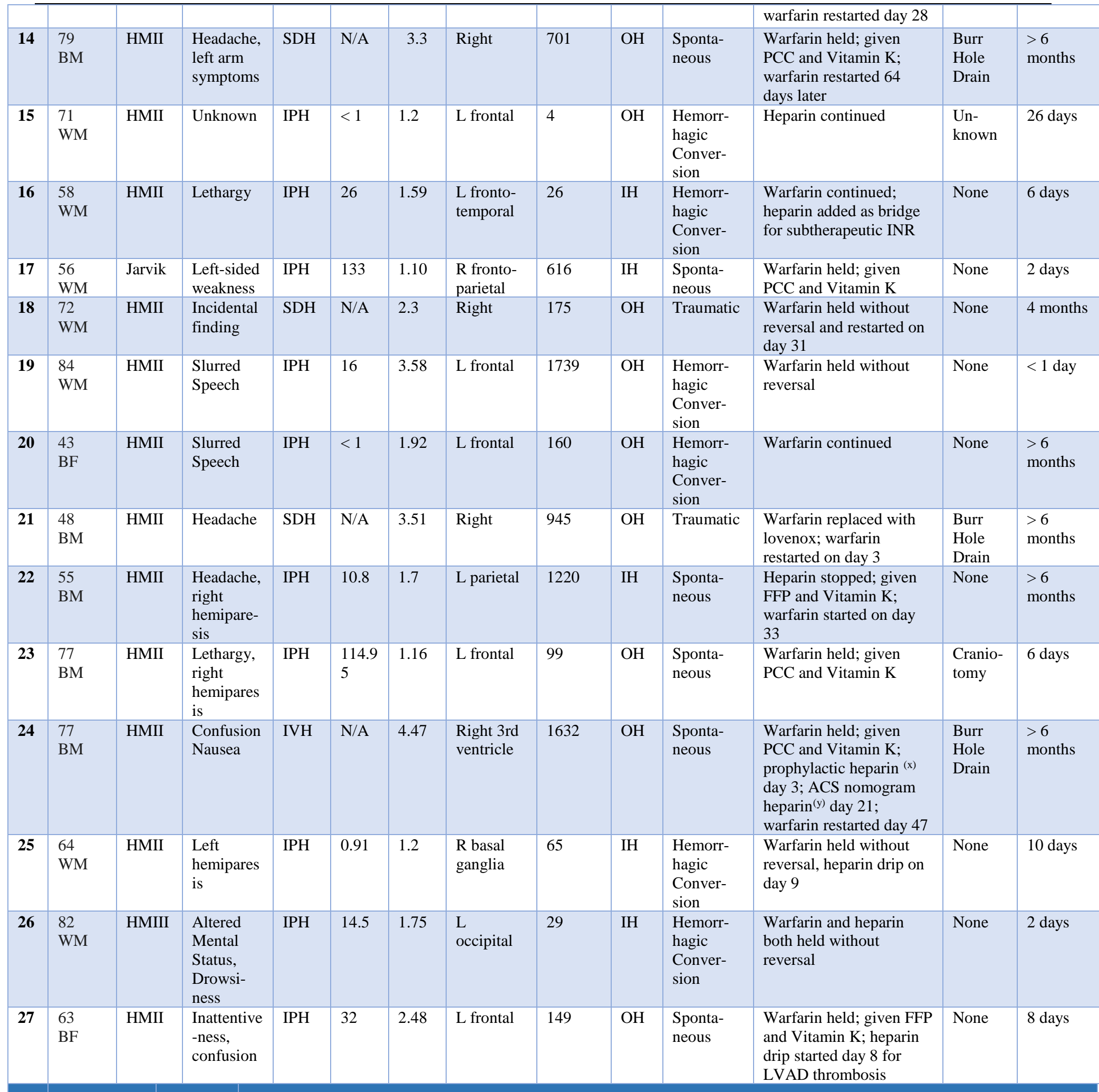


$\mathrm{ADL}=$ above detectable limit; $\mathrm{BF}=$ black female, $\mathrm{BM}=$ black male; HMII=HeartMate II; HMIII= HeartMate III; IH= in hospital; IPH=intraparenchymal hemorrhage; NAF= Native American Female; $\mathrm{OH}=$ out of hospital; $\mathrm{PCC}=$ prothrombin complex concentrate; $\mathrm{SDH}=$ subdural hematoma; $\mathrm{WF}=$ white female; $\mathrm{WM}=$ white male

(x) 5000 units unfractionated heparin every 8 hours ${ }^{(y)}$ unfractionated heparin infusion titrated to goal heparin correlation of $0.3-0.5$.

\section{ICH Characteristics}

Among the 27 cases of $\mathrm{ICH}$, there were 17 intraparenchymal, 7 subdural, 2 subarachnoid, and 1 intraventricular event. Of the 17 patients who developed IPH, 10 presented with spontaneous hemorrhage, 6 with hemorrhagic conversion of an ischemic stroke, and 1 with head trauma. Of the 7 patients who suffered SDH, 6 had a history of head trauma or fall while 1 had no history of either. Of the 2 patients who developed SAH and 1 with IVH, none had a history of recent head trauma, fall, or ischemic stroke. Thirteen patients developed an ICH while inpatient, and of these 4 had been hospitalized for LVAD implantation, 4 were being treated for bacteremia or LVAD pocket infection, 2 for ischemic stroke, 1 for CHF exacerbation, 1 for pre-operative anti-coagulation management, and 1 for suspected LVAD thrombosis. Fourteen patients developed bleeding outside of the hospital (Table 1).

\section{Anti-thrombotic Management \& Complications}

Twenty-one patients (78\%) were on antiplatelet therapy at presentation with 19 $(70 \%)$ on aspirin alone, one (4\%) on prasugrel alone, and one $(4 \%)$ on a combination of aspirin and clopidegrel. Only 4 patients had antiplatelet therapy continued at the time of bleed, all of whom were being treated for hemorrhagic conversion of an ischemic stroke. Three patients had antiplatelet therapy restarted at 1 week, 1 month, and 2 months respectively.

Twenty-five patients (93\%) were anticoagulated at presentation with 19 (70\%) on warfarin, $2(7 \%)$ on a heparin infusion, and $4(15 \%)$ on warfarin plus a heparin infusion. INR at time of presentation ranged from 1.1 to above the detectable limit. Due to recent history of Gl bleeding, 2 patients (7\%) were not anticoagulated at the time of their $\mathrm{ICH}$. Among the 25 patients who were anticoagulated, 13 were managed with immediate cessation of anticoagulation and administration of reversal products (Group A), 7 were managed with immediate cessation of anticoagulation without administration of reversal products (Group B), and 5 were managed with continuation of anticoagulation (Group C). In Group A, reversal products utilized included Fresh Frozen Plasma (FFP), Prothrombin Complex Concentrate (PCC), vitamin K, and protamine. In Group B, many different clinical factors contributed to the decision to withhold reversal products. Specifically, 4 patients presented with unrecoverable bleeds and were managed with comfort measures only, 1 patient had an asymptomatic SDH for which reversal was deemed unnecessary, 1 patient had an ischemic stroke with a very small ICH from hemorrhagic conversion, and 1 patient had signs of chronic subclinical LVAD thrombosis. Given the poor prognosis of this group in general, their follow-up time was very limited. In Group C, anticoagulation was continued for several reasons. 
Three patients had an ischemic or embolic stroke with two of the three having less than $1 \mathrm{~cm}^{3}$ volume $\mathrm{ICH}, 1$ patient had a SDH with intact neurologic status, and 1 patient had a history of antiphospholipid antibody syndrome complicated by prior LVAD thrombosis. In these 5 cases it was thought that the risk of discontinuing anticoagulation outweighed the benefits.

With a median follow-up of 26 days (range $=1-1350$ days) in these 25 patients with $\mathrm{ICH}$ while on anticoagulation, there were a total of 2 patients with subsequent device thrombosis and 1 patient with ischemic stroke over the course of the study - none of which occurred within the first week after bleed. Group A had a median of 6 days off anticoagulation and 60 days median follow-up time with only 1 (8\%) patient developing device thrombosis at day 8 . One (8\%) patient in this group developed ischemic stroke at day 14. Four (31\%) had evidence of worsening bleeding which all occurred within 1 week (Figure 1). Group B had a median of 2 days off anticoagulation and 2 days of follow up time with no episodes of device thrombosis or ischemic stroke. One (14\%) patient had evidence of worsening bleeding on day 1. Group C had a median follow-up time of 330 days with $1(20 \%)$ device thrombosis at day 5 in a patient with a history of antiphospholipid antibody syndrome and multiple LVAD thromboses in the past. Expansion of ICH occurred in $1(20 \%)$ patient on day 27 (Table 2 ).

\section{Care Team Designation \& Surgical Intervention}

In all cases, an interdisciplinary discussion took place between cardiology, neurosurgery, neurology, cardiac surgery, and/or hematology. Eight (30\%) patients had at least one consultant note that weighed the risk of device thrombosis heavily in clinical decision-making. Eighteen (67\%) patients were treated in the cardiac intensive care unit (ICU), $2(7 \%)$ in the neurosurgical ICU, and $6(22 \%)$ in the cardiac intermediate unit (Table 3). One patient was taken to the operating room and died before being assigned to a hospital unit. Glasgow Coma Scale (GCS) was recorded only in 12 (44\%) patients. NIH Stroke Scale (NIHSS) was recorded for 8 patients (30\%) and Glasgow Outcome Scale (GOS) was not recorded for any patients.

Table 2: LVAD Thrombosis, Ischemic Stroke, Worsening Bleed, and Death Stratified by Anticoagulation Strategy

\begin{tabular}{|c|c|c|c|}
\hline & \multicolumn{3}{|c|}{ Anticoagulation Management } \\
\hline & Group A & Group B & Group C \\
\hline & Reversed & Held & Continued \\
\hline No. of patients & 13 & 7 & 5 \\
\hline $\begin{array}{l}\text { Follow up time (days), } \\
\text { median }\end{array}$ & 60 & 2 & 330 \\
\hline Days off $A C$, median $^{(a)}$ & 6 & 2 & - \\
\hline Days off $A C$, range $e^{(a)}$ & $1-65$ & $1-31$ & - \\
\hline Device Thrombosis & $1(8)$ & $0(0)$ & $1(20)$ \\
\hline Ischemic Stroke & $1(8)$ & $0(0)$ & $0(0)$ \\
\hline Expansion of $/ \mathrm{CH}^{(b)}$ & $4(31)$ & $1(14)$ & $1(20)$ \\
\hline Death at 24 hours (\%) & $2(15)$ & $3(43)$ & $0(0)$ \\
\hline Death at 30 days (\%) & $6(46)$ & $6(86)$ & $2(40)$ \\
\hline Death at 6 months (\%) & $8(62)$ & $7(100)$ & $2(40)$ \\
\hline
\end{tabular}


(a) Ended with either resumption of anticoagulation or death

(b) defined as clinical or radiographic evidence of hemorrhage expansion

$\mathrm{AC}=$ Anticoagulation

$\mathrm{ICH}=$ Intracranial hemorrhage

Table 3: Care Team and Hospital Unit

\begin{tabular}{|c|c|}
\hline \multirow{2}{*}{\multicolumn{2}{|c|}{ No. of Patients, total (a) }} \\
\hline & \\
\hline Cardiology & $21(78)$ \\
\hline Cardiac Surgery & $2(7)$ \\
\hline Neurosurgery & $3(11)$ \\
\hline Consultants (\%) (b) & \\
\hline Neurosurgery & $20(74)$ \\
\hline Neurology & $14(52)$ \\
\hline Cardiology & $5(19)$ \\
\hline Hematology & $2(7)$ \\
\hline Hospital unit (a)(\%) & \\
\hline CICU & $18(67)$ \\
\hline $\mathrm{NSICU}$ & $2(7)$ \\
\hline $\begin{array}{l}\text { Cardiac Intermediate } \\
\text { Care }\end{array}$ & $6(22)$ \\
\hline
\end{tabular}

(a) One patient was taken to the OR and died before being assigned to a unit and before consult teams could be assigned

Surgical intervention was performed in a total of 6 patients. This included 4 patients with SDH who underwent Burr hole drainage and 2 patients with IPH who underwent open craniotomy.

\section{Outcomes}

Survival outcomes varied by management group (A, B, and C) and by ICH subtype (Table 2 and 4, respectively). Thirty-day mortality was $46 \%, 86 \%$, and $40 \%$ in Groups A, B, and C respectively, while 6-month mortality was $62 \%, 100 \%$, and $40 \%$ respectively. Patients who presented with IPH had the worst outcomes with a $76 \%$ rate of death at 30 days compared to only $14 \%, 0 \%$, and $0 \%$ in patients with $\mathrm{SDH}, \mathrm{SAH}$, and IVH respectively. Both patients who underwent open craniotomy for IPH died within 6 days. On the other hand, all four patients who underwent Burr hole drainage survived to 6 months. 
The VAD Journal: The journal of mechanical assisted circulation and heart failure

Table 4: Survival Outcomes

\begin{tabular}{|c|c|c|c|c|c|}
\hline & IPH & SDH & SAH & IVH & All \\
\hline Death at 24 hours (\%) & $4(24)$ & $1(14)$ & $0(0)$ & $0(0)$ & $5(19)$ \\
\hline Death at 30 days (\%) & $13(76)$ & $1(14)$ & $0(0)$ & $0(0)$ & $14(52)$ \\
\hline Death at 6 months (\%) & $14(82)$ & $3(42)$ & $1(50)$ & $0(0)$ & $18(72)$ \\
\hline
\end{tabular}

$\mathrm{IPH}=$ intraparenchymal hemorrhage; $\mathrm{IVH}=$ intraventricular hemorrhage; $\mathrm{SAH}=$ subarachnoid hemorrhage; SDH=subdural hemorrhage

\section{Discussion}

In this case series assessing anticoagulation management, surgical intervention, care team utilization, complications, and outcomes among continuous-flow LVAD patients presenting with $\mathrm{ICH}$, we found that the majority of patients were middleaged males with a Heartmate II device, almost all of whom were anticoagulated at presentation. The etiology of ICH included traumatic, spontaneous, and hemorrhagic conversion with about half of patients developing $\mathrm{ICH}$ while already in the hospital for an alternative reason. Clinical management strategies varied across our patient population with mixed outcomes. Most notably, cessation of anticoagulation with administration of reversal products was generally well tolerated in the short term, with no episodes of device thrombosis or ischemic stroke occurring within the first week when this strategy was employed. On the other hand, about one-quarter of patients in the study had clinical or radiographic evidence of worsening bleeding; the majority of which did occur within the first week. Intracranial hemorrhage was associated with poor outcomes as only onethird of patients in the study survived to 6 months.

While the management of anticoagulation in the LVAD patient with ICH remains a unique and challenging dilemma, the data currently available to guide treatment remains limited. In a single-center study of LVAD patients presenting with $\mathrm{ICH}$, Wilson et al followed 22 individuals who had warfarin held and reversed for a median of 10.5 days and found that none developed device thrombosis or stroke (10). This study, however, was performed largely among patients supported with a pulsatile LVAD rather than a contemporary continuous-flow device. A similar study by Wong et al analyzed the efficacy of vitamin $\mathrm{K}$ antagonist reversal using 4-factor PCC versus traditional reversal strategies in continuous flow devices and found only one LVAD thrombosis and no ischemic strokes among 20 cases (11). This study was also limited by the fact that the average time off anticoagulation was not reported, making it difficult to interpret and generalize. In the largest observational study to date, Tahir et al found that ICH size, midline shift, and GCS score were predictive of mortality, but they did not report data on reversal strategies or rates of device thrombosis (12). Our study revealed no device thrombosis or ischemic stroke within the first week in patients managed with swift cessation and reversal of anticoagulation. However, almost one-third of patients had consult notes which weighed the risk of device thrombosis and ischemic stroke heavily in clinical decision-making. Additionally, a significant proportion of patients across all treatment groups demonstrated clinical or radiographic evidence of worsening 
bleeding within the first week. One could therefore make a compelling argument that in the short term, the risk of bleeding may outweigh the risk of clotting in such cases. With the fully magnetically-levitated HeartMate 3 demonstrating lower rates of ischemic stroke and pump thrombosis but similar rates of bleeding compared to the older HeartMate 2, we expect this pattern may persist $(13,14)$.

The role of neurosurgical intervention in this patient population remains even more unclear. Although small studies suggest non-cardiac surgery in the LVAD patient does not significantly increase morbidity and mortality, very few specifically assess outcomes after intracranial hematoma evacuation (15-17). In a recent study by Ikeda et al, 6 of 7 LVAD patients with ICH who underwent acute operative intervention ( 6 craniotomies and 1 burr hole drainage) died within 30 days (18). Wilson et al reported similar results, with four of five patients who underwent craniotomy dying within 60 days (10). At our institution, both patients treated with open craniotomy died within 1 week while all 4 patients who underwent Burr hole drainage for subdural hematoma survived to 6 months. Other studies have also shown Burr hole drainage to be generally well tolerated in these cases (10). Whether the poor outcomes seen with craniotomy are due to sicker patients going to the $\mathrm{OR}$ is unclear, and more data is needed to make definitive recommendations. Notably, the 2015 American Heart Association/American Stroke Association guidelines on the management of spontaneous intracranial hemorrhage state that, in general, the role of surgery for supratentorial $\mathrm{ICH}$ is not well established (19).

Our study is the first to describe care team designation and hospital unit admission patterns as key factors that may impact patient management. The majority of patients were treated in either a cardiac ICU or a cardiac intermediate care unit with cardiac intensivists or cardiac surgeons serving as leaders of the care team. Two patients were managed in the neurosurgical ICU. With very few exceptions, all patients were also followed by a neurosurgery and/or neurology consultant team. Whether to manage these patients in the cardiac versus neurosurgical ICU remains controversial, with clinical resources and specialized nurse training playing a key role in this debate. The cardiac ICU allows for closer and more experienced monitoring of potential LVAD dysfunction such as device thrombosis, arrhythmia, and heart failure, whereas the neurosurgical ICU may allow for more optimal management of $\mathrm{ICH}$ including monitoring of intracranial pressures, tracking of cerebral perfusion pressures, early identification of dynamic changes in neurologic function, and other tasks that may benefit from more specialized nursing care (20). As an example, we found that less than half of our patients who were treated in a cardiac unit had either Glasgow Coma Scale (GCS) or NIH Stroke Scale (NIHSS) recorded during their hospital stay, and none of the patients had a Glasgow Outcome Scale documented. Given that GCS and other stroke assessment scores have been shown to be important in the management of $\mathrm{ICH}$, and that the AHA/ASA recommends that nurses caring for these patients be trained in obtaining these scores, this study highlights the need to more closely examine these care practices in our cardiac-specific ICUs (19). Given the low risk of early device thrombosis in our patient population, the potential for rapid neurologic decline, and the level of specialized care needed to treat an acute intracranial bleed, one might consider treating LVAD patients with $\mathrm{ICH}$ in a neurosurgical ICU or dedicated stroke unit rather than the cardiac ICU. The risks 
and benefits of this approach should currently be evaluated on a case-by-case basis, but we believe this strategy could and should be more rigorously assessed in a prospective clinical study.

In addition to prospective clinical assessment, we suggest that more data elements concerning ICH be collected as part of the INTERMACS database. This should include ICH subtypes, management practices (including pharmacologic reversal agent use and operative interventions), and neuro-specific outcomes. More data is certainly needed to understand which components of management truly influence patient outcomes.

Our study utilized rigorous chart review to gather clinical data not previously reported in larger studies, and is the first to collect data on care team designation and hospital unit following $\mathrm{ICH}$. There are, however, several limitations that should be considered. First, given the small sample size our results should be viewed as largely descriptive. We did not have the necessary power to make direct statistical comparisons across groups. Instead we focused on providing rigorous data related to care processes and stroke characteristics that we hope will serve as a basis for future investigation. Second, the majority of patients in Group B suffered devastating bleeds, were transitioned to comfort measures within 1 or 2 days of admission, and died shortly thereafter, thus limiting their follow up time. Finally, as previously mentioned, our records did not consistently include data relevant to stroke outcomes such as GCS, NIHSS, GOS, and Rankin scale.

\section{Conclusion}

Patients with LVADs who experience an $\mathrm{ICH}$ have very high mortality rates. Their care is multidisciplinary and can involve operative intervention in certain circumstances. Early reversal of anticoagulation is generally well tolerated with a low risk of early device thrombosis or ischemic stroke. Like for many hemorrhagic complications of LVADs, bleeding after ICH often persists or worsens. Additional prospective studies are needed to provide future guidance to clinicians faced with this difficult, and often devastating clinical event. 


\section{References}

1. Bui AL, Horwich TB, and Fonarow GC. Epidemiology and risk profile of heart failure. Nature Reviews Cardiology. 2011;8:30-41.

2. Rose EA, Gelijns AC, Moskowitz AJ, et al. Long-term Use of a Left Ventricular Assist Device for End-Stage Heart Failure. New England Journal of Medicine. 2001;345:1435-43.

3. Frazier $\mathrm{OH}$, Rose $\mathrm{EA}, \mathrm{Oz} \mathrm{MC}$, et al. Multicenter clinical evalutation of the HeartMate vented electric left ventricular assist system in patient awaiting heart transplantation. Journal of Thoracic and Cardiovascular Surgery. 2001;122:1186-95.

4. Netuka I, Sood P, Pya Y, et al. Fully Magnetically Levitated Left Ventricular Assist System for Treating Advanced HF. Journal of the American College of Cardiology. 2015;66:2579-89.

5. Kirklin JK, Pagani FD, Kormos RL, et al. Eight annual INTERMACS report: Special focus on framing the impact of adverse events. The Journal of Heart and Lung Transplantation. 2017;36:1080-86.

6. Acharya D, Loyaga-Rendon R, Morgan CJ, et al. INTERMACS Analysis of Stroke during Support with Continuous Flow Left Ventricular Assist Devices: Risk factors and Outcomes. Journal of the American College of Cardiology: Heart Failure. 2017;5:703-11.

7. Kato TS, Schulze PC, Yang J, et al. Pre-operative and post-operative risk factors associated with neurologic complications in patient with advanced heart failure supported by a left ventricular assist device. Journal of Heart and Lung Transplantation. 2012;31:1-8.

8. Tsukui H, Abla A, Teuteberg JJ, et al. Cerbrovascular accidents in patients with a ventricular assist device. Journal of Thoracic and Cardiovascular Surgery. 2007;114-23.

9. Kormos RL, Cowger J, Pagani FD, et al. The Society of Thoracic Surgeons Intermacs database annual report: Evolving indications, outcomes, and scientific partnerships. The Journal of Heart and Lung Transplantation. 2019;38:114-26.

10. Wilson TJ, Stetler WR, Al-Holou WN, Sullivan SE, and Fletcher JJ. Management of intracranial hemorrhage in patients with left ventricular assist devices. Journal of Neurosurgery. 2013;118:1063-8.

11. Wong JK, Chen PC, Falvey J, et al. Anticoagulation Reversal Strategies for Left Ventricular Assist Device Patients Presenting with Acute Intracranial Hemorrhage. ASAIO Journal 2016;62:552-7.

12. Tahir RA, Rotman LE, Davis MC, et al. Intracranial Hemorrhage in Patients with a Left Ventricular Assist Device. World Neurosurgery. 2018;113:e71421. 
13. Mehra MR, Naka Y, Uriel N, et al. A Fully Magnetically Levitated Circulatory Pump for Advanced Heart Failure. New England Journal of Medicine. 2017;376:440-50.

14. Mehra MR, Goldstein DJ, Uriel N, et al. Two-Year Outcomes with a Magnetically Levitated Cardiac Pump in Heart Failure. New England Journal of Medicine. 2018;378:1386-95.

15. Barbara DW, Wetzel DR, Pulido JN, et al. The perioperative management of patients with left ventricular assist devices undergoing noncardiac surgery. Mayo Clinic Proceedings. 203;88:674-82.

16. Bhat G, Kumar S, Aggarwal A, et al. Experience With Noncardiac Surgery in Destination Therapy Left Ventricular Assist Devices Patients. ASAIO Journal. 2012;4:396-401.

17. Ahmed M, Le H, Aranda JM, and Klodell CT. Elective noncardiac surgery in patients with left ventricular assist devices. Journal of Cardiac Surgery. 2012;27:639-42.

18. Ikeda DS, Bansal S, Shaw A, et al. Acute Operative Intervention for Intracranial Hemorrhage in Adult Patients with Ventricular Assist Device Therapy Associated with Fatal Outcomes. International Journal of Surgery Research and Practice. 2014;1:1-5.

19. Hemphill JC $3^{\text {rd }}$, Greenberg SM, Anderson CS, et al. Guidelines for the Management of Spontaneous Intracerebral Hemorrhage: A Guideline for Healthcare Professionals From the American Heart Association/American Stroke Association. Stroke. 2015;46:2032-60.

20. Alberts MJ, Latchar RE, Selman WR, et al. Recommendations for comprehensive stroke centers: a consensus statement from the Brain Attack Coalition. Stroke. 2005;36:1597-616. 